\title{
THE FAMILY OF ANCILLARY STATISTICS
}

\author{
By D. BASU \\ Indian Statistical Institute, Calcutta
}

SUMMARY. Though the marginal distributions of the ancillary statistics are independent of the parameter they are not useless or informationless. A set of ancillaries may sometimes summarise the whole of the information contained in the sample. A classification of the ancillaries in terms of the partial order of their information content is attempted here. In general there are many maximal ancillaries. Among the minimal ancillaries there exists a unique largest one. When there exists a complete sufficient statistic, the problem of tracking down the maximal and minimal ancillaries becomes greatiy simplified.

\section{INTRODUCTION}

An ancillary ${ }^{1}$ statistic is one whose distribution is the same for all possible values of the unknown parameter. A statistic that is not ancillary may be called 'informative'. The classical example of an ancillary statistic is the following:

Example (a): Let $X$ and $Y$ be two positive valued random variables with the joint density function

$$
f(x, y)=e^{-\theta x-\frac{y}{\theta}}, x>0, y>0, \theta>0 .
$$

Here $F=X Y$ is an ancillary statistic. The maximum likelihood estimator $T=\sqrt{Y} / \bar{X}$ of $\theta$ is not a sufficient statistic. However, the pair $(F, T)$ is jointly. sufficient.

The above example shows that though an ancillary statistic, by itself, fails to provide any information about the parameter, yet in conjunction with another statistic--which, as we shall presently see, need not be informative-may supply valuable information ${ }^{2}$ about the parameter. In the following example we have given a family of ancillary statistics that are jointly equivalent to the whole sample.

Example (b) : Let $X$ and $Y$ be independent normal variables with unknown means $\theta$ and unit standard deviations. Here $X-Y$ is an ancillary statistic. It is commonly believed that every ancillary statistic (in this situation) is necessarily a function of $X-Y$. That, however, is not true.

Let

$$
F_{c}=F_{c}(X, Y)=\left\{\begin{array}{lll}
X-Y & \text { if } \quad X+Y<c \\
Y-X & \text { if } \quad X+Y \geqslant c
\end{array}\right.
$$

where $c$ is a fixed constant.

1. The name 'ancillary' is due to Fisher (1925). The name 'distribution-free' is also in use and perhaps would have been more appropriate in the present context.

2. See Fisher (1956) for a discussion of how the aneillary information may (according to Fisher) be recovered. 
Since $X-Y$ and $Y-X$ are identically distributed and each is independent of $X+Y$ it at once follows that $F_{c}$ is independent of $X+Y$ and has the same distribution as that of $X-Y$. Thus, $F_{c}$ is ancillary for each $c$. Consider now the family $\left\{\boldsymbol{F}_{c}\right\},-\infty<c<\infty$, of ancillary statistics. For fixed $X$ and $Y$ the different values of $F_{c}$ (for varying $c$ ) are either $X-Y$ or $Y-X$. The value $c_{0}$ of $c$ where $F_{c}$ changes sign $\left(F_{c}\right.$ does not change sign only if $X-Y=0$ and that is a null event) is the value of $X+Y$. Thus, given $F_{c}(X, Y)$ for all $c$ we can find $X+Y$ and $X-Y$. Hence, the family $\left\{F_{c}\right\}$ of ancillary statistics is equivalent to the whole sample $(X, Y)$. The countable family $\left\{F_{c}\right\}$ where $c$ runs through the set of rational numbers is easily seen to be also equivalent to $(X, Y)$.

The author (Basu ; 1955, 1958) has shown that, under very mild restrictions, any statistic independent of a sufficient statistic is ancillary and that the converse proposition is also true, provided the sufficient statistic is complete.

In Example (b) the statistic $T=X+Y$ is a complete sufficient statistic. A statistic $F$ can, therefore, be ancillary if and only if $F$ is independent of $T$. The following is a general method for constructing statistics independent of $T$. Start with any ancillary statistic $F$. In general, there will be many measure-preserving transformations of $F$ (i.e. a mapping $\varphi$ of the range space of $F$ into itself such that $\varphi(F)$ and $F$ are identically distributed). For each real $t$, define a measure-preserving transformation $\varphi_{t}$ of $F$. Then, take the statistic $\varphi_{T}(F)$. Subject to some measurability restrictions, $\varphi_{T}(F)$ will be independent of $T$ and hence will be ancillary. In Example (b) we took $F=X-Y$ and $\varphi_{t}(F)=F$ or $-F$ according as $t<c$ or $\geqslant c$.

If a statistic $F$ is ancillary then every (measurable) function of $F$ is also ancillary. The statistic $F_{2}$ is said to include (or be more informative than) the statistic $F_{1}$ if $F_{1}$ can be expressed as a function of $F_{2}$. In this case we write $F_{2} \supset F_{1}$ or $F_{1} \subset F_{2}$. Two statistics are said to be equivalent if each can be expressed as a function of the other.

Example (c) : Let $X_{1}, X_{2}, \ldots X_{n}$ be $n$ independent observations on a normal variable with mean $\theta$ and s.d. unity. Then each of the $n-1$ statistics

$$
F_{1}=X_{1}-X_{2}, F_{2}=\left(X_{1}-X_{2}, X_{1}-X_{3}\right), \ldots F_{n-1}=\left(X_{1}-X_{2}, X_{1}-X_{3}, \ldots X_{1}-X_{n}\right)
$$

is ancillary and

$$
F_{1} \subset F_{2} \subset \ldots \subset F_{n-1}
$$

The two ancillary statistics $F_{n-1}$ and $F=\left(X_{2}-X_{1}, X_{2}-X_{3}, \ldots X_{2}-X_{n}\right)$ are easily seen to be equivalent.

From Example(b) it is obvious that $F_{n-1}$ does not include all ancillary statistics.

An ancillary statistic $M$ is said to be 'maximal' if there exists no non-equivalent ancillary $M^{*}$ such that $M \subset M^{*}$. Thus, given any ancillary $F$, either it is maximal or there exists an ancillary $F^{*} \supset F$. Given any ancillary $F_{0}$, there exists (Theorem 2) 


\section{THE FAMILY OF ANCILLARY STATISTICS}

a maximal ancillary $M \supset F_{0}$. In general there exists many non-equivalent maximal ancillaries. A typical property (Cor. to Theorem 4) of a maximal ancillary $M$ is that, for any ancillary $F$ not included in $M$, the pair $(M, F)$ is informative.

A minimal ancillary is one that is included in every maximal ancillary. Among the class of minimal ancillaries there exists (Theorem 5) a unique largest one $G_{0}$. In the absence of a better name we prefer to call $G_{0}$ the laminal ancillary. $G_{0}$ includes every minimal ancillary and is included in every maximal ancillary. A typical property (Theorem 6) of a minimal ancillary $G$ is that, for any ancillary $F$, the pair $(G, F)$ is ancillary.

If there exists a complete sufficient statistic $G$, then, any ancillary statistic $F$, such that the pair $(G, F)$ is essentially equivalent to the whole sample, is shown (Theorem 7) to be essentially maximal. Under some further restrictions, the laminal ancillary is shown (Theorem 8) to be essentially equivalent to a constant.

In the following sections we elaborate on the above sketch of the family-tree of ancillary statistics. For the sake of elegance and brevity of exposition we use the language of sub $\sigma$-fields. Reference may be made to Bahadur $(1954,1955)$ for excellent expositions of the sub $\sigma$-field approach.

\section{Definitions}

Let $(\mathcal{X}, \mathcal{B})$ be an arbitrary measurable space and let $\left\{P_{\theta}\right\}, \theta \in \Omega$ be a family of probability measures on $\mathbb{B}$. Any statistic $T$ induces a sub $\sigma$-field $\mathcal{B}_{T} \subset \mathbb{B}$. Instead of dealing with statistics it is more convenient (in the present context) to deal with the corresponding sub $\sigma$-fields.

Definition 1: The event $A \in \mathscr{B}$ is said to be ancillary if $P_{\theta}(A)$ is the same for all $\theta \in \Omega$. The family of all ancillary events is denoted by $\mathcal{A}$.

It is easy to check that the family $\mathcal{A}$ is closed for complementation and countable disjoint unions. However, in general $\mathcal{A}$ is not closed for intersection (i.e. $\mathcal{A}$ is not a $\sigma$-field).

In order to show that the family $\mathcal{A}$ in Example (b) do not constitute a $\sigma$-field, we have only to check that

$$
\begin{aligned}
P_{\theta}\left[X-Y>0 \text { and } F_{c}(X, Y)>0\right] & =P_{\theta}(X-Y>0 \text { and } X+Y<c) \\
& =\frac{1}{2} \int_{-\infty}^{c} \frac{1}{\sqrt{4 \pi}} e^{-\frac{1}{4}(x-2 \theta)^{2}} d x
\end{aligned}
$$

which varies with $\theta$.

In Example (b) the Borel-extension of $\mathcal{A}$ is $\mathscr{B}$. 
Example (d): Let $\mathscr{X}$ consist of the three points $a, b$ and $c$ and let the corresponding probability measures be $\frac{1}{4}-\theta, \frac{1}{2}$, and $\frac{1}{4}+\theta$ respectively, where $0<\theta<\frac{1}{4}$. Here $\mathcal{A}$ consists of the four sets $\phi,[b],[a, c]$ and $\mathscr{X}$ and so $\mathcal{A}$ is a sub $\sigma$-field of $\mathscr{B}$.

Definition 2: A $\sigma$-field $\mathcal{F}$ is said to be ancillary if $\mathscr{F} \subset \mathcal{A}$. A $\sigma$-field that is not ancillary is called informative.

A statistic is ancillary or informative according as the corresponding $\sigma$-field is so.

Definition 3: Two ancillary sets $A$ and $B$ are said to conform if $A B$ is also ancillary. If $A$ conforms to $B$ then we write $A \sim B$. Since $P_{\theta}(A B)+P_{\theta}\left(A B^{\prime}\right)$ $=P_{\theta}(A)$ it follows that $A \sim B$ if and only if $A \sim B^{\prime}$.

If $A$ conforms to every one of a sequence of disjoint sets $B_{1}, B_{2} \ldots$ then it is easy to check that $A \sim \bigcup B_{i}$.

Definition 4: Let $\Gamma_{0}$ be the family of all ancillary sets $B$ such that $B \sim A$ for all $A \in \mathcal{A}$.

Clearly $\phi$ and $\mathscr{X}$ belong to $\Gamma_{0}$. From what we have said before it follows that $\Gamma_{0}$ is closed for complementation and countable disjoint unions.

Theorem 1: The family $\Gamma_{0}$ is a $\sigma$-field.

Proof: It is enough to show that $\Gamma_{0}$ is closed for intersection. Let $B_{1}$ and $B_{2}$ both belong to $\Gamma_{0}$ and let $A \in \mathscr{A}$. From $B_{2} \in \Gamma_{0}$ it follows that $B_{2} A \in \mathcal{A}$. From $B_{1} \in \Gamma_{0}$ it then follows that $B_{1} B_{2} A \in \mathcal{A}$. Since $A$ is an arbitrary ancillary set, it follows that $B_{1} B_{2} \in \Gamma_{0}$.

We shall later on see that the ancillary $\sigma$-field $\Gamma_{0}$ corresponds to the laminal ancillary $G_{0}$ that we have referred to in $\S 1$.

The family $\mathcal{A}$ of ancillary sets is a $\sigma$-field if and only if every pair of ancillary sets conform to one another, i.e. if $\mathcal{A}=\Gamma_{0}$.

Example (e): Let $\mathscr{X}$ consist of the five points $a, b, c, d$ and $e$ with the corresponding probabilities $\frac{1}{2}, \theta, \theta, \frac{1}{4}-\theta$ and $\frac{1}{4}-\theta$ respectively, where $0<\theta<\frac{1}{4}$. In this case $\Gamma_{0}$ consists of the four sets $\phi,[\mathrm{a}],[b, c, d, e]$, and $\mathscr{X}$. The two sets $[b, d]$ and $[b, e]$ are both ancillary but they do not conform. Here $\mathcal{A}$ is wider than $\Gamma_{0}$ and is not a $\sigma$-field.

Definition 5: The ancillary $\sigma$-field $\mathscr{F}_{2}$ is said to include the ancillary $\sigma$-field $\mathscr{F}_{1}$ (in symbols $\mathscr{F}_{2} \supset \mathscr{F}_{1}$ or $\mathscr{F}_{1} \subset \mathscr{F}_{2}$ ) if every element of $\mathscr{F}_{1}$ is an element of $\mathscr{F}_{2}$.

The above partial order on ancillary $\sigma$-fields corresponds to the inclusion relationship for ancillary statistics.

Definition 6: The ancillary $\sigma$-field $\mathcal{M}$ is said to be maximal if there exists no other ancillary $\sigma$-field $\mathscr{N}^{*}$ such that $\mathscr{N}^{*} \supset \mathscr{K}$. 


\section{THE FAMILY OF ANCILLARY STATISTICS}

Definition 7 : The intersection of all the maximal ancillary $\sigma$-fields is called the laminal ancillary.

The laminal ancillary is the largest ancillary that is included in all maximal ancillaries.

\section{Existence AND CHARACTERIZATIONS OF MAXIMAL AND LAMINAL ANCILLARIES}

The following theorem is fundamental.

Theorem 2: Given any ancillary $\sigma$-field $\mathscr{F}_{0}$ there exists a maximal ancillary $\sigma$-field $\mathscr{M} \supset \mathscr{F}_{0}$.

Proof: We first prove that given any family $\left\{\boldsymbol{F}_{j}\right\}, j \in J$ of ancillary $\sigma$-fields that are linearly ordered (by the inclusion relationship), the Borel-extension $\mathscr{F}$ of $\cup \mathscr{F}_{j}$ is also ancillary.

Clearly, $\bigcup \mathcal{F}_{j}$ contains $\phi$ and $\mathscr{X}$ and is closed for complementation. Since $\left\{\boldsymbol{F}_{j}\right\}$ is linearly ordered it follows that $\bigcup \mathcal{F}_{j}$ is also closed for finite unions. That is, $\bigcup \mathcal{F}_{j}$ is a field of sets.

Since each $\mathcal{F}_{j}$ is ancillary, the restriction of $P_{\theta}$ to $\bigcup \mathcal{F}_{j}$ is a measure $Q$ that does not depend on $\theta$. From the fundamental Extension Theorem of measures (Kolmogorov, 1933) we know that the extension of $Q$ to $\mathcal{F}$ is unique.

It follows at once that the restriction of $P_{\theta}$ to $\mathcal{F}$ is the same for all $\theta$, i.e. $\mathscr{F}$ is an ancillary $\sigma$-field.

Now let $\mathcal{C}$ be the family of all ancillary $\sigma$-fields that include $\mathcal{F}_{0}$. Since corresponding to any linearly ordered sub-family of $\mathcal{C}$ there exists an ancillary $\sigma$-field that includes every member of the sub-family it follows from Zorn's Lemma that $\mathcal{C}$ has a maximal element.

Let $\left\{\mathscr{M}_{i}\right\}, i \in I$ be the family of all maximal ancillary $\sigma$-fields. We at once have the

Theorem 3: $\mathscr{A}=\bigcup \mathscr{N}_{i}$

Proof: We have only to note that corresponding to any element $A$ of $\mathcal{A}$ there exists an ancillary $\sigma$-field that contains $A$ as an element and then apply Theorem 2.

Corollary : If $\left\{\mathscr{M}_{i}\right\}$ consists of only one $\sigma$-field $\mathscr{M}_{0}$ then $\mathscr{A}=\mathscr{M}_{0}=\Gamma_{0}$.

Thus, in any situation where there are non-conforming ancillary sets, the family $\left\{\mathscr{K}_{i}\right\}$ has at least two members.

In Example (d) there is a unique maximal ancillary. In Example (e) there are exactly two maximal ancillaries namely :

and

$$
\mathscr{M}_{1}=\text { the } \sigma \text {-field spanned by }[a] \text { and }[b, d]
$$$$
\mathscr{\varkappa}_{2}=\text { the } \sigma \text {-field spanned by }[a] \text { and }[b, e] \text {. }
$$

Theorem 4: If the ancillary set $A$ does not belong to the maximal ancillary $\mathcal{N}$ then $A$ does not conform to at least one element of $\mathcal{M}$. 
Proof: Suppose on the contrary that $A$ cnforms to every element of $\mathscr{M}$. Consider the family $\mathscr{N}^{*}$ of sets $A X \cup A^{\prime} Y$ where $X$ and $Y$ are arbitrary elements of $\mathscr{M}$. Clearly $\mathscr{M} \subset \mathscr{N}^{*}$ but not conversely.

Since

$$
\left(A X \cup A^{\prime} Y\right)^{\prime}=A X^{\prime} \cup A^{\prime} Y^{\prime},
$$

and

and

$$
\bigcup\left(A X_{i} \cup A^{\prime} Y_{i}\right)=A\left(\bigcup X_{i}\right) \cup A^{\prime}\left(\bigcup Y_{i}\right)
$$

$$
P_{\theta}\left(A X \cup A^{\prime} Y\right)=P_{\theta}(A X)+P_{\theta}\left(A^{\prime} Y\right),
$$

it follows that $\mathscr{N}^{*}$ is also an ancillary $\sigma$-field.

This, however, contradicts the maximality of $\mathscr{N}$.

Corollary : If $\mathcal{H}$ be any maximal ancillary and if the ancillary $\sigma$-field $\mathscr{F}$ is not included in $\mathscr{M}$ then the smallest $\sigma$-field containing both $\mathscr{M}$ and $\mathcal{F}$ is informative.

Theorem 5: $\mathscr{M}_{i}=\Gamma_{0}$

Proof : Since every element of $\Gamma_{0}$ conforms (by definition) to every ancillary event, if follows from Theorem 4 that $\Gamma_{0} \subset \mathscr{N}_{i}$ for all $i$, i.e. $\Gamma_{0} \subset\left(\cap \mathscr{M}_{i}\right)$.

Now let $B \in \bigcap \mathscr{N}_{i}$ and $A$ be an arbitrary ancillary set. From Theorem 3 it follows that $A \in \mathscr{M}_{i}$ for some $i$.

Hence $B$ and $A$ are together as elements of some $\mathscr{M}_{i}$ and so $B \sim A$.

Since $A$ is arbitrary it follows that $B \in \Gamma_{0}$.

$\therefore\left(\cap \mathscr{N}_{i}\right) \subset \Gamma_{0}$ and so the equality is proved.

Theorem 6: For any ancillary $\sigma$-field $\mathcal{F}$ the smallest $\sigma$-field containing both $\mathcal{F}$ and $\Gamma_{0}$ is also ancillary.

Proof: Consider the family of 'rectangular' sets $X \cap Y$ where $X \epsilon \mathcal{F}$ and $Y \in \Gamma_{0}$. From the definition of $\Gamma_{0}$ it follows that all such sets are ancillary and that they conform to one another. The family of sets that may be formed by finite unions of rectangular sets form a field of sets and each of them is ancillary. The rest follows from the Extension Theorem of Measures.

\section{When a COMPLETE SUFFICIENT STATISTIC EXISTS}

In general there exist many maximal ancillaries. For instance, in Example (b) there are uncountably many maximal ancillaries. In order to see this, let us consider the family $\left\{A_{c}\right\}$ of ancillary events where $A_{c}=\left\{(X, Y) \mid F_{c}(X, Y)>0\right.$. If $c<d$, then

$$
\begin{aligned}
P_{\theta}\left(A_{c} A_{d}\right) & =P_{\theta}(X-Y>0 \text { and } X+Y<c)+P_{\theta}(Y-X>0 \text { and } X+Y \geqslant d) \\
& \fallingdotseq \frac{1}{2}\left[1-\int_{c}^{d} \frac{1}{2 \sqrt{\pi}} e^{-\frac{1}{4}(x-2 \theta)^{2}} d x\right]
\end{aligned}
$$

which varies with $\theta$.

Thus, the members of the family $\left\{A_{c}\right\}$ of ancillary sets are mutually nonconforming. Hence the maximal ancillaries including the different members of the family are all different. 


\section{THE FAMILY OF ANCILLARY STATISTICS}

Though there may exist many maximal ancillaries, it is not, in general, easy to prove the maximality of a particular ancillary. However, in the situations where we have a complete sufficient statistic, it is rather easy to demonstrate the maximality of a large class of ancillaries.

The following property of complete sufficient statistics is useful. ${ }^{1}$ Here we state and prove the result in terms of $\sigma$-fields.

Lemma (Basu, 1955): If $\mathcal{G} \subset \mathbb{B}$ be a boundedly complete sufficient $\sigma$-field and $A$ any ancillary event, then $A$ is independent of $\mathcal{G}$.

Proof: Let $\varphi=P(A \mid \mathcal{G})$ be the conditional probability of $A$ given $\mathcal{G}$. That is, $\varphi$ is a $\mathcal{G}$-measurable function such that

$$
P_{\theta}(A G)=\int_{\theta} \varphi d P_{\theta} \text { for all } \theta \epsilon \Omega \text { and } G \epsilon \mathcal{G} \text {. }
$$

Since $\mathcal{G}$ is sufficient, it follows that $\varphi$ may be chosen to be independent of $\theta$. Also the set of $x$ 's for which $\varphi(x)$ lies outside the interval $(0,1)$ is of zero-measure for each $\theta \in \Omega$.

Taking $G=\mathscr{C}$ we have

$$
P_{\theta}(A)=\int_{\mathscr{C}} \varphi d P_{\theta} \text { for all } \theta \epsilon \Omega .
$$

Since $P_{\theta}(A)$ is independent of $\theta$ and $\varphi$ is $\mathcal{G}$-measurable, it follows from the bounded completeness of $\mathcal{G}$ that $\varphi=P_{\theta}(A)$ almost surely for all $\theta \epsilon \Omega$.

$$
\begin{aligned}
\quad P_{\theta}(A G) & =\int_{G} \varphi d P_{\theta} \\
& =P_{\theta}(A) P_{\theta}(G) \text { for all } \theta \epsilon \Omega \text { and } G \in \mathcal{G} .
\end{aligned}
$$

That is, $A$ is independent of all $G \in \mathcal{G}$

Before proceeding further we need a slightly wider definition of maximality for an ancillary $\sigma$-field.

Definition 8: The two $\mathcal{B}$-measurable sets $A$ and $B$ are said to be essentially equal if

$$
\begin{aligned}
P_{\theta}(A \Delta B) & \equiv P_{\theta}\left(A B^{\prime} \cup A^{\prime} B\right) \\
& =0 \quad \text { for all } \theta \in \Omega .
\end{aligned}
$$

Definition 9 : Two sub $\sigma$-fields $\mathcal{F}_{1}$ and $\mathcal{F}_{2}$ are said to be essentially equivalent if corresponding to any set belonging to one of them there exists an essentially equal set belonging to the other.

Definition 10: Any ancillary $\sigma$-field that is essentially equivalent to a maximal ancillary is called essentially maximal.

Theorem 7 : If $\mathcal{G}$ be a boundedly complete sufficient $\sigma$-field then any ancillary $\mathcal{F}$ such that the Borel-extension of $\mathcal{G} \cup \mathcal{F}$ is essentially equivalent to $\mathbb{B}$, is essentially maximal.

1 See Basu (1955) and Hogg and Craig (1956) for some other interesting applications. 
Vol. 21] SANKHYA : THE INDIAN JOURNAL OF STATISTICS [ PARTS 3 \& 4

Proof: Let $\mathcal{M}$ be a maximal ancillary including $\mathcal{F}$ and let $M$ be an arbitrary element of $\mathcal{H}$. For proving the essential maximality of $\mathscr{F}$ we have to establish the existence of an $F_{0} \in \mathscr{F}$ such that $F_{0}$ is essentially equal to $M$.

Let $\mathfrak{B}^{*}$ be the Borel extension of $\mathscr{F} \cup \mathcal{G}$. Since $\mathfrak{B}^{*}$ is essentially equivalent to $\mathbb{B}$, there exists an $M^{*} \in \mathbb{B}^{*}$ such that $M^{*}$ is essentially equal to $M$.

Since $M \in \mathscr{M} \supset \mathscr{F}$ and $M^{*}$ is essentially equal to $M$, it follows that $M^{*}$ is an ancillary set conforming to every $F \in \mathcal{F}$. Clearly, the two measures $P$ and $Q$ on $\mathcal{F}$, defined by the relations $P(F)=P_{\theta}(F)$ and $Q(F)=P_{\theta}(M * F)$, are both independent of $\theta$.

Therefore, the conditional probability function

$$
\varphi=P_{\theta}\left(M^{*} \mid \mathcal{F}\right)=\frac{d Q}{d P}
$$

is independent of $\theta$.

Thus, $\varphi$ is an $\mathcal{F}$-measurable function on $\mathscr{X}$ such that

$$
P_{\theta}\left(M^{*} F\right)=\int_{F} \varphi d P_{\theta} \text { for all } \theta \in \Omega \text { and } F \in \mathcal{F} .
$$

Let $F$ and $G$ be typical elements of $\mathscr{F}$ and $\mathcal{G}$ respectively. Since $\mathscr{F}$ is ancillary and $\mathcal{G}$ is boundedly complete sufficient, it follows (from the Lemma) that $\mathscr{F}$ and $\mathcal{G}$ are independent.

$$
\begin{aligned}
& \int_{F G} \varphi d P_{\theta}=\int_{\mathscr{X}}\left(\varphi \mathscr{X}_{F}\right) \mathscr{X}_{G} d P_{\theta} \quad\left(\mathscr{X}_{F} \text { and } \mathscr{X}_{G}\right. \text { are characteristic func- } \\
&\text { tions of } F \text { and } G)
\end{aligned}
$$

Again, since $M^{*} \sim F$ it follows (from the Lemma) that $M^{*} F$ is independent of $G$.

$$
\begin{aligned}
& \therefore \quad \int_{F G} \mathscr{X}_{M^{*}} d P_{\theta}=P_{\theta}\left(M^{*} F G\right)=P_{\theta}\left(M^{*} F\right) P_{\theta}(G) . \\
& \text { From }(\alpha) \text { and }(\beta) \text { we have } \\
& \qquad \int_{F G}\left(\varphi-\mathscr{X}_{M^{*}}\right) d P_{\theta}=0 \text { for all } F \in \mathcal{F} \text { and } G \in \mathcal{G} .
\end{aligned}
$$

Since $\varphi-\mathscr{X}_{M^{*}}$ is $\mathcal{B}^{*}$-measurable it at once follows that

$$
\int_{B}\left(\varphi-\mathscr{X}_{M^{*}}\right) d P_{\theta}=0 \text { for all } B \in \mathbb{B}^{*}
$$

Therefore, for each $\theta \epsilon \Omega, \varphi(x)-\mathscr{X}_{M^{*}}(x)=0$ for almost all $x$.

Let $F_{0}=\{x \mid \varphi(x)=1\}$. Clearly $F_{0} \in \mathscr{F}$ and is essentially equal to $M^{*}$.

Since $M^{*}$ is essentially equal to $M$ the Theorem is proved. 


\section{THE FAMILY OF ANCILLARY STATISTICS}

In Example (b), $X+Y$ is a complete sufficient statistic. Also for any fixed $c$, the pair $\left(X+Y, F_{c}\right)$ is equivalent to the sample $(X, Y)$. Hence it follows that every $F_{c}$ is an essentially maximal ancillary. In Example (c), the ancillary $F_{n-1}$ together with the complete sufficient statistic $X_{1}+X_{2}+\ldots+X_{n}$ is equivalent to the whole sample and, therefore, is essentially maximal. A large number of similar situations are covered by Theorem 7 .

Having partially settled the question of maximal ancillaries let us turn our attention to the laminal ancillary.

The laminal ancillary is the largest ancillary $\sigma$-field that is included in all maximal ancillaries. From Theorem 5 we have that the class $\Gamma_{0}$ of ancillary sets $C$ that conform to every ancillary set is the laminal ancillary.

Let $\Lambda$ be the family of sets that are essentially equal to either the empty set $\phi$ or the whole space $\mathscr{C}$. That is, $\Lambda$ is the family of all sets $E$ such that $P_{\theta}(E)$ is either $\equiv 0$ or $\equiv 1$ for all $\theta \epsilon \Omega$. It is easy to check that $\Lambda$ is a $\sigma$-field and that $\Lambda \subset \Gamma_{0}$. The following theorem covers a number of important cases.

Theorem 8: If the following conditions are satisfied then $\Gamma_{0}=\Lambda$.

i) $\mathcal{F}$ is an essentially maximal ancillary.

ii) There exists an informative set $G$ which is independent of $\mathcal{F}$.

iii) For every $F \in \mathcal{F}$ such that $0<P_{\theta}(F)<1$ there exists $F^{*} \in \mathscr{F}$ such that $P_{\theta}\left(F^{*}\right)=P_{\theta}(F)$ and $P_{\theta}\left(F F^{*}\right)<P_{\theta}(F)$.

Proof: Let $C$ be an arbitrary element of $\Gamma_{0}$. We have to prove that $P_{\theta}(C)$ $=0$ or 1 . If possible let $0<P_{\theta}(C)<1$.

Now, $\mathcal{F}$ is essentially equivalent to a maximal ancillary and $C$ belongs to every maximal ancillary. Hence, there exists $F \in \mathcal{F}$ which is essentially equal to $C$. Thus, $F$ conforms to every ancillary set and $0<P_{\theta}(F)<1$.

Let $G$ and $F^{*}$ satisfy conditions (ii) and (iii) respectively and let $A=G F \cup G^{\prime} F^{*}$. Since $G$ is independent of $\mathcal{F}$, we have

$$
\begin{aligned}
P_{\theta}(A) & =P_{\theta}(G) P_{\theta}(F)+P_{\theta}\left(G^{\prime}\right) P_{\theta}\left(F^{*}\right) \\
& =P_{\theta}(F)\left[P_{\theta}(G)+P_{\theta}\left(G^{\prime}\right)\right] \\
& =P_{\theta}(F) .
\end{aligned}
$$

That is, $A$ is an ancillary set.

Now

and, therefore,

$$
A F=G F \cup G^{\prime}\left(F F^{*}\right)
$$

$$
\begin{aligned}
P_{\theta}(A F) & =P_{\theta}(G) P_{\theta}(F)+P_{\theta}\left(G^{\prime}\right) P_{\theta}\left(F F^{*}\right) \\
& =P_{\theta}\left(F F^{*}\right)+P_{\theta}(G)\left[P_{\theta}(F)-P_{\theta}\left(F F^{*}\right)\right] .
\end{aligned}
$$

Let us note that $P_{\theta}\left(F F^{*}\right)$ and $P_{\theta}(F)-P_{\theta}\left(F^{*} F^{*}\right)$ are both independent of $\theta$ and that the latter is not zero. Again since $G$ is informative $P_{\theta}(G)$ is not independent of $\theta$. Hence $A F$ is informative, which is a contradiction. Therefore, $P_{\theta}(C)=0$ or 1 , i.e. $C \epsilon \Lambda$, which proves the theorem. 
Vol. 21] SANKHYA : THE INDIAN JOURNAL OF STATISTICS [ PARTs $3 \& 4$

If the conditions of Theorem 7 are satisfied then $\mathcal{F}$ and any informative $G \in \mathcal{G}$ satisfies conditions (i) and (ii) of Theorem 8 . We have then only to check whether condition (iii) is satisfied or not. If the restriction of $P_{\theta}$ to $\mathcal{F}$ be non-atomic then it is very easy to see that condition (iii) is also satisfied.

In Examples (b) and (c) the (essentially) maximal ancillaries have continuous (non-atomic) distributions and so Theorem 8 holds. Most of the familiar cases where a complete sufficient statistic exists fall under the above category.

Example (f) : Let $X$ be a single observation on a normal variable with mean zero and standard deviation $\sigma$. Here $X^{2}$ is a complete sufficient statistic.

$$
\text { Let } Y=\left\{\begin{aligned}
-1 & \text { of } X<0 \\
1 & \text { if } X \geqslant 0
\end{aligned}\right.
$$

Here the pair $\left(Y, X^{2}\right)$ is equivalent to the whole sample $X$.

$\therefore \quad Y$ is an essentially maximal ancillary.

The sub $\sigma$-field generated by $Y$ consists of the four sets $\phi,(-\infty, 0),[0, \infty)$ and $\mathcal{F}$. Condition (iii) of Theorem 8 is clearly satisfied. Therefore, the laminal ancillary $\Gamma_{0}$ is the same as $\Lambda$.

\section{ACKNOWLEDGement}

I wish to thank Dr. R. R. Bahadur for some useful discussions.

\section{References}

BAHADUR, R. R. (1954): Sufficiency and statistical decision functions. Ann. Math. Stat., 25, 423.

(1955): Statistics and subfields. Ann. Math. Stat., 26, 490.

BASU, D. (1955) : On statistics independent of a complete sufficient statistics. Sankhyā, $15,377$.

(1958): On statistics independent of a sufficient statistic. Sankhyā, 20, 223.

FIsher, R. A. (1925) : Theory of statistical estimation. Proc. Camb. Phil. Soc., 22, 700.

(1956): Statistical Methods and Scientific Inference, Oliver and Boyd, London.

Hogg, R. V. and Cratg, A. T. (1956) : Sufficient statistics in elementary distribution theory. Sankhya $17,209$.

Kolmogorov, A. N. (1933): Foundations of The Theory of Probability, Chelsea Publishing Company, New York.

Paper received: July, 1959. 